\title{
A comprehensive analysis of antigen-specific autoimmune liver disease related autoantibodies in patients with multiple sclerosis
}

\author{
Zisis Tsouris ${ }^{1}$, Christos Liaskos ${ }^{2}$, Efthymios Dardiotis ${ }^{1}$, Thomas Scheper ${ }^{3}$, Vana Tsimourtou' ${ }^{1}$, Wolfgang Meyer ${ }^{3}$, \\ George Hadjigeorgiou ${ }^{1,4}$ and Dimitrios P. Bogdanos ${ }^{2 *}$
}

\begin{abstract}
Introduction: Abnormal liver function tests are frequently seen in patients with multiple sclerosis (MS) and their origin at times is attributed to the possible co-occurrence or the de novo induction of autoimmune liver diseases (AILD), namely autoimmune hepatitis (AIH) and primary biliary cholangitis (PBC), but comprehensive analysis of AlLD-related autoantibody has not been carried out.

Aim: To assess the presence of AILD-related autoantibodies in a well-defined cohort of MS patients, and to assess their clinical significance.

Materials and methods: 133 MS (93 female) patients (102 RRMS, 27 SPMS, and 5 PPMS), mean age $42.7 \pm 11.9$ SD years, mean duration of disease $11.2 \pm 7.2$ years were studied. 150 age and sex-matched healthy individuals were tested as normal controls (NCs).Autoantibody testing was performed by indirect immunofluorescence (IF) using triple tissue and HEp-2, a multiparametric line immunoassay detecting anti-LKM1 (anti-CYP2D6), anti-LC1 (anti-FTCD), soluble liver antigen/liver-pancreas(anti-SLA/LP), AMA-M2, and AMA-MIT3 (BPO), PBC-specific ANA (anti-gp210, anti-sp100 and anti-PML), and ELISA for anti-F-actin SMA and anti-dsDNA antibodies,
\end{abstract}

Results: Reactivity to at least one autoantibody was more frequent in MS patients compared to NCs (30/133, 22.6\% vs $12 / 150,8 \%)$ NCs $(p=0.00058)$. SMAs by IIF were more frequent in MS patients $(18 / 133,13.53 \%)$ compared to NCs $(6 / 150,4 \%, p=0.002 \%)$. The AlH-1 related anti-F-actin SMA by ELISA were present in $21(15.8 \%)$, at relatively low titres (all but three of the SMA-VG pattern by IF); anti-dsDNA in 3 (2.3\%), and anti-SLA/LP in none; AlH-2 anti-LKM1 autoantibodies in $1(0.8 \%$, negative by IF), and anti-LC1 in none; PBC-specific AMA-M2 in $2(1.5 \%$, both negative for AMA-MIT3 and AMA by IF) and PBC-specific ANA anti-PML in 6 (4.5\%), anti-sp100 in $1(0.8 \%)$ and anti-gp210 in 1 (0.8\%). Amongst the 30 MS patients with at least one autoantibody positivity, only 4 (3\%) had overt AlLD (2 AlH-1 and 2 PBC). Autoantibody positivity did not differ between naïve MS patients and patients under treatment.

${ }^{*}$ Correspondence: bogdanos@med.uth.gr

${ }^{\dagger}$ Zisis Tsouris, Christos Liaskos and Efthymios Dardiotis first authorship

2 Department of Rheumatology and Clinical Immunology, University

General Hospital of Larissa, Faculty of Medicine, School of Health

Sciences, University of Thessaly, Biopolis, Larissa 40500, Greece

Full list of author information is available at the end of the article

(c) The Author(s) 2020. This article is licensed under a Creative Commons Attribution 4.0 International License, which permits use, sharing, adaptation, distribution and reproduction in any medium or format, as long as you give appropriate credit to the original author(s) and the source, provide a link to the Creative Commons licence, and indicate if changes were made The images or other third party material in this article are included in the article's Creative Commons licence, unless indicated otherwise in a credit line to the material. If material is not included in the article's Creative Commons licence and your intended use is not permitted by statutory regulation or exceeds the permitted use, you will need to obtain permission directly from the copyright holder. To view a copy of this licence, visit http://creativeco mmons.org/licenses/by/4.0/. 
Conclusions: Despite the relatively frequent presence of liver autoantibodies, tested either by IF or molecular assays, overt AILD is rather infrequent discouraging autoantibody screening strategies of MS patients in the absence of clinical suspicion.

Keywords: ANA, Autoantibody, Autoimmunity, Autoimmune hepatitis, Autoimmune rheumatic disease, Druginduced liver injury, Liver diseases

\section{Introduction}

Multiple sclerosis (MS) is an autoimmune demyelinating disease, frequently characterized by concurrent autoimmune diseases, mainly including autoimmune thyroid disease, both Hashimoto's thyroiditis and Graves' disease, autoimmune rheumatic diseases, insulin-dependent diabetes mellitus, idiopathic Addison's disease, atrophic gastritis, myasthenia gravis and inflammatory bowel disease [1-4]. It becomes apparent that in view of these con-current autoimmune diseases, several MS-unrelated autoantibodies have so far been reported.

Autoimmune liver diseases (AlLDs) appear less frequently, though reports suggest their prevalence being significantly higher to large populations of untreated MS compared to the general population [5-7]. Interferonbeta, used to treat MS of both the re- lapsing-remitting (RRMS) and of the secondary progressive form (SPMS), the two most common clinical phenotypes of MS, has been considered a usual trigger of autoantibodies, and at times of overt autoimmune disease [8]. In particular, sporadic cases of autoimmune hepatitis $(\mathrm{AIH})$ have been described in untreated MS patients or following treatment with interferon-beta or other immunomodulatory therapeutic agents, including steroids and glatiramer acetate [9]. Despite the slightly increased prevalence of $\mathrm{AIH}$, compared to that of the general population, routine screening for AILD of patients with established MS has been discouraged and is only recommended in those with abnormal liver enzymes or patients with a clinical suspicion of liver disease of unknown origin and drug-induced liver injury $[5,10]$.

It is not yet clear, whether AILD-related autoantibodies are de novo induced by MS-related immunomodulatory agents, pre-exist in the context of concurrent sub-clinical or clinical AILD or both [11-18].

Meticulous assessment of AILD-related autoantibodies $[19,20]$, paying attention to humoral responses against their molecular targets, in consecutive series of MS patients has not yet been performed.

The aim of the present study was to provide a complete profiling of these autoantibodies in patients from a single referral centre for MS patients in Central Greece, as this could initiate the impetus for assessing the diagnostic and clinical significance of AILD-related autoantibodies in this disease, providing novel insights as to whether routine autoab testing is needed for proper clinical decision making.

\section{Materials and methods \\ Material}

The study included 133 consecutive MS (93 female) patients (102 RRMS, 27 SPMS, and 4 PPMS-Primary Progressive MS), mean age $42.7 \pm 11.9$ SD years, mean duration of disease $11.2 \pm 7.2$ years. Table 1 shows the major clinical and laboratory findings of MS patients. Thirty patients (22.6\%) were naïve (untreated) and 103 (77.4\%) were on treatment including 35 patients $(26.3 \%$ of the total) on interferon- $\beta$ and 68 patients (51.1\% of the total) on other immunomodulatory agents (24 patients with natalizumab; 20 with fingolimod; 16 with glatiramer; 5 with teriflunomide; and 3 patients with mitoxantrone).

One hundred and fifty serum samples from healthy individuals tested as normal controls were also studied.

The study was been approved by the local Ethics Committee of the University General Hospital of Larissa, University of Thessaly (4/15-04-2016). Written informed consent was obtained by all participants.

Table 1 Major clinical and laboratory characteristics of patients with multiple sclerosis included to the study

\begin{tabular}{ll}
\hline & $(\mathbf{n}=\mathbf{1 3 3})$ \\
\hline Sex (M/F) (\%) & $40(30.1 \%) / 93(69.9 \%)$ \\
Age (years) (mean \pm SD) & $42.7 \pm 11.9$ \\
Age at diagnosis (years) (mean \pm SD) & $31.5 \pm 10.4$ \\
(>20/<20 years) & $132(99.2 \%) / 1(0.8 \%)$ \\
(>30/<30 years) & $110(82.7 \%) / 23(17.3 \%)$ \\
(>40/<40 years) & $72(51.4 \%) / 61(45.9 \%)$ \\
(>50/<50 years) & $37(27.8 \%) / 96(72.2 \%)$ \\
(>60/<60 years) & $14(10.5 \%) / 119(89.5 \%)$ \\
(>70/<70 years) & $1(0.8 \%) / 132(99.2 \%)$ \\
Type of MS (RRMS/SPMS/PPMS)(\%) & $102(76.6 \%) / 27(20.3 \%) / 4(3 \%)$ \\
Duration (years) (mean \pm SD) & $11.2 \pm 7.2$ \\
EDSS score & $3.3 \pm 2.1$ \\
Number of relapses & $5 \pm 3.6$ \\
Progression index & $0.42 \pm 0.56$ \\
\hline
\end{tabular}

EDSS: Expanded Disability Status Scale score, RRMS: Relapsing-remitting Multiple Sclerosis, SPMS: Secondary Progressive Multiple Sclerosis, PPMS: Primary Progressive Multiple Sclerosis 


\section{Methods}

\section{Autoantibody testing}

Autoantibody testing was performed by conventional indirect immunofluorescence (IIF) using triple liver kidney stomach tissue (cut off for positivity: 1/40) and HEp-2(cut off for positivity: 1/160). Reports for AILDrelated autoantibodies by IIF included ANA of any pattern, SMA of any pattern (actin-SMA in liver tissue, vessels/glomeruli/tubuli in kidney tissue, AMA, antiliver kidney microsomal (anti-LKM), and anti-liver cytosol (anti-LC).

A multiparametric line immunoassay detecting antiLKM1(anti-CYP2D6), anti-LC1(anti-FTCD), soluble liver antigen/liver-pancreas(anti-SLA/LP), AMA-M2, and AMA-MIT3, PBC-specific ANA (anti-gp210, anti-sp100 and anti-PML) and anti-Ro52 was used as a molecular-based assay for the detection of AILDrelated autoantibodies.

AIH-related SMA directed against F-actin were tested by an ELISA (Inova, San Diego, CA, USA), according to the manufacturer's instructions [19]. All sera were tested at 1/101 dilution per manufacturers directives and according to our previously reported protocols [19].

Anti-ssDNA and anti-dsDNA antibodies testing was performed by ELISA, as per manufacturer's instructions (Inova Diagnostics, San Diego, CA, USA), in accordance to the instructions of the manufacturer.

\section{Statistical analysis}

All data are reported as percentages (\%). Serum levels variation in each patients group was defined by mean and standard deviation (SD). Differences in categorical data between groups were tested by two-tailed Pearson's Chi square and Fisher's Exact Test after correction for continuity. Differences in numerical data between groups were tested by the two-tailed Student's $t$ test. $p$-values smaller than 0.05 were considered significant. All statistical calculations were performed with IBM SPSS Statistics 20 software.

\section{Results}

Overall, 30/133 (22.6\%) MS patients had at least one of the tested autoantibody specificities compared to 12 (8\%) NCs $(\mathrm{p}=0.00058)$.

ANAs by IIF were present in $8(6.01 \%)$ patients with MS compared to $3(2 \%) \mathrm{NCs}(\mathrm{p}=\mathrm{ns})$. The median titre of MS positive samples was $1 / 160$ by IIF on HEp-2 ( 2 were positive at $1 / 80 ; 6$ at $1 / 160$ and 2 at a $1 / 320$ dilution). Concerning IIF patterns, 4 had an homogenous pattern, 3 had fine speckled pattern and 1 one had multiple nuclear dots pattern (the same patient was also anti-sp100 positive, which is compatible with PBC-specific ANAs).
SMAs by IIF were more frequent in MS patients $(18 / 133,13.53 \%)$ compared to NCs $(6 / 150,4 \%$, $\mathrm{p}=0.002 \%)$. Using the line liver blotting assay the following autoantibody's reactivities were observed: AMA-MIT3 in $0 \mathrm{MS}$ compared to $0 \mathrm{NCs}(\mathrm{p}=1.00)$; AMA-M2 in $2(1.5 \%)$ compared to $0 \mathrm{NCs}(\mathrm{p}=\mathrm{ns})$ (none of whom was positive for AMA-BPO by the same line immunoassay or by IIF); PBC-specific ANA anti-gp210 in $1(0.8 \%)$ MS compared to $1(0.7 \%) \mathrm{NCs}(\mathrm{p}=\mathrm{ns})$; PBCspecific ANA anti-sp100 in $1(0.8 \%)$ MS compared to $0(0 \%) \mathrm{NCs}(\mathrm{p}=\mathrm{ns})$; PBC-specific ANA anti-PML in 6 (4.5\%) MS compared to $1(0.7 \%) \mathrm{NCs}(\mathrm{p}=0.053)$; antiAIH2-specific anti-LKM1 (anti-CYP2D6) in $1 \%(0.8 \%$, negative by IIF) MS compared to $0 \% \mathrm{NCs}(\mathrm{p}=\mathrm{ns})$; anti-LC1 (anti-FTCD) in 0\% MS compared to 0\% NCs ( $\mathrm{p}=\mathrm{ns})$. AIH-specific anti-SLA/LP in 0\% MS compared to $0 \% \mathrm{NCs}(\mathrm{p}=\mathrm{ns})$; and, anti-Ro52 in 2 (1.5\%) MS compared to $2(1.3 \%) \mathrm{NCs}(\mathrm{p}=\mathrm{ns})$. Representative cases of antibodies detection are showed in Fig. 1.

By ELISA, AIH-1-specific anti-F-actin were more frequent in patients with MS compared to NCs $(21 / 133$, $15.8 \%$ vs $7 / 150,4.7 \%, p=0.0017$ ) (all but three of them were tested positive for the SMA-VG pattern by IIF, amongst them 3 patients were also positive for SMA-F pattern by IIF); anti-ssDNA in 13 (9.77\%) MS compared to $5(3.33 \%) \% \mathrm{NCs}(\mathrm{p}=\mathrm{ns})$; and anti-dsDNA in 3

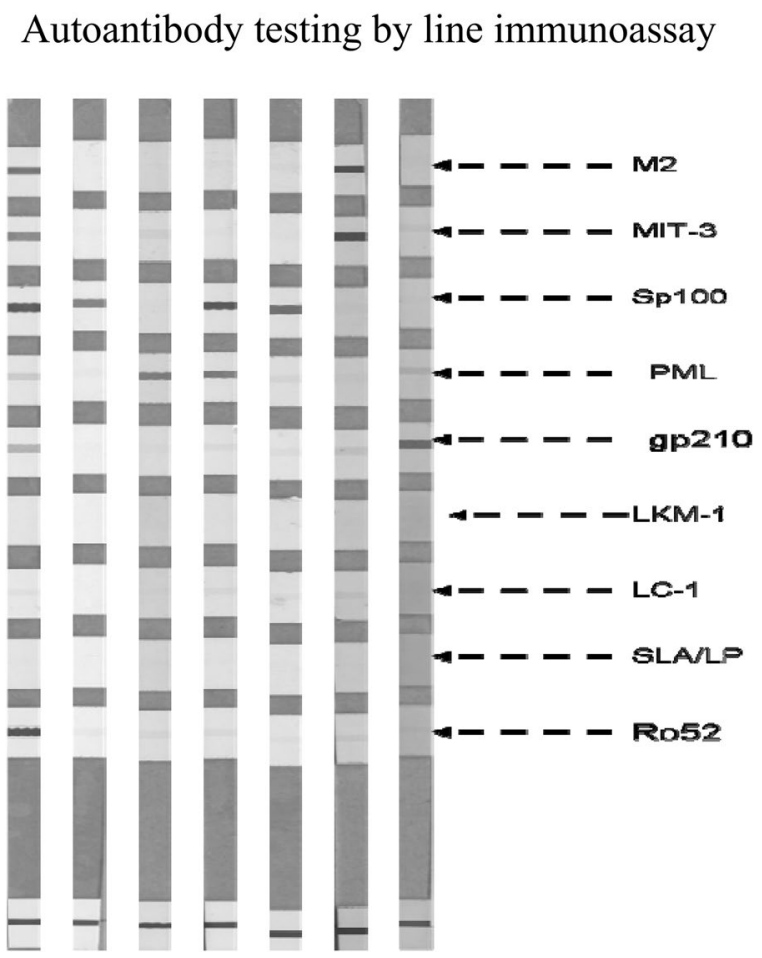

Fig. 1 Representative cases of abs detection in patients with MS using a line immunoassay 
(2.3\%) MS patients compared to $2(1.33 \%) \mathrm{NCs}(\mathrm{p}=\mathrm{ns})$, all three were also positive for anti-ssDNA. Levels of anti-ssDNA, anti-dsDNA and anti-Factin antibodies in patients with MS are showed in Fig. 2. The magnitudes of autoantibodies responses against $\mathrm{F}$-actin did not differ between MS patients and NCs $(13.53 \pm 10.62$ vs $11.81 \pm 17.8 \mathrm{RU} / \mathrm{ml} \mathrm{p}=\mathrm{ns}$, Fig. 3).

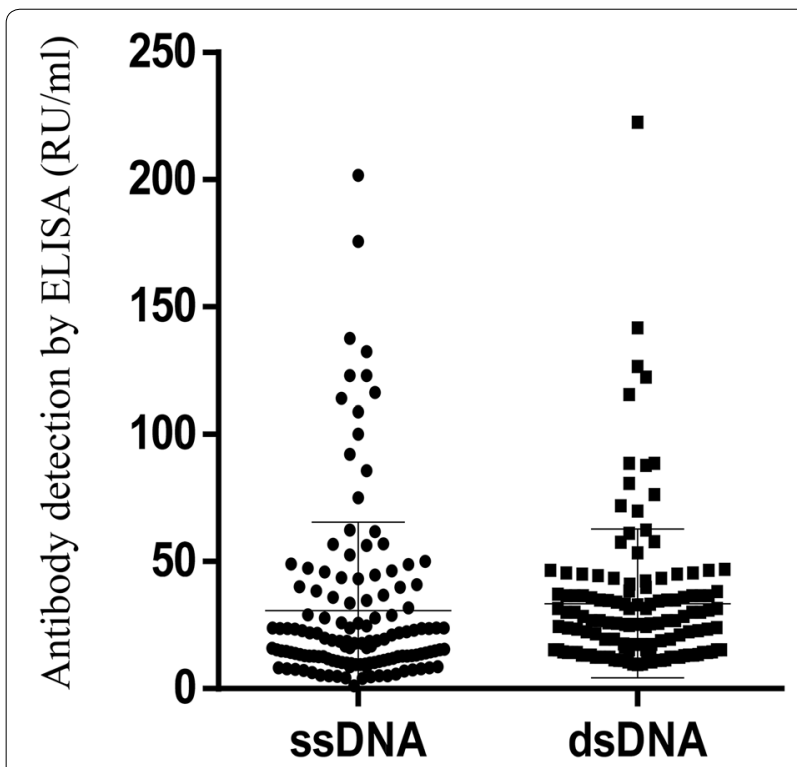

Fig. 2 Levels of anti-ssDNA and anti-dsDNA and anti-F-actin abs in patients with multiple sclerosis
Of the $30 \mathrm{MS}$ patients with at least one AILD-related autoantibody positivity only $2(2 / 30,6.7 \%)$ had overt AILD ( 1 with a known diagnosis of AIH-1 and 1 with a known diagnosis of $\mathrm{PBC}$ ). One additional patient with detectable AMA and anti-gp210 antibodies in our cohort had also elevated liver enzymes and a diagnosis of PBC were confirmed. Eight of the 30 patients with MS and detectable AILD-related autoantibodies were followed up (median 73 months, range 39-126 months) and retesting of available serum samples was performed to witness the over time behavior of autoantibodies. Also, the biochemical and clinical profile of these patients that would potentially place a suspicion of a chronic liver disease, including AILD, was recorded. All patients who were tested had normal liver functions test during the period of follow up, with the exception of one female patient, tested positive for anti-F-actin, who showed transient mild elevation of transaminases, approximately 2 years after the date of experiment. Autoantibody positivity did not differ between naïve MS patients and patients under treatment: out of the 30 naïve MS patients, $8(26.7 \%)$ had at least one autoantibody compared to $22 / 103$ (21.4\%, $\mathrm{p}=\mathrm{ns})$ MS under treatment. Regarding the most frequent autoantibody specificities, SMAs by IIF were present in $6 / 30(20 \%)$ naïve MS patients compared to $12 / 103$ (11.7\%) under treatment $(\mathrm{p}=\mathrm{ns})$.

AIH-1-specific anti-F-actin (by ELISA) were present in 6/30 (20\%) naïve MS compared to $15 / 103(14.6 \%)$ MS under treatment $(\mathrm{p}=\mathrm{ns})$. No other association was found between autoantibody positivity and treatment status or type of MS-related treatment.

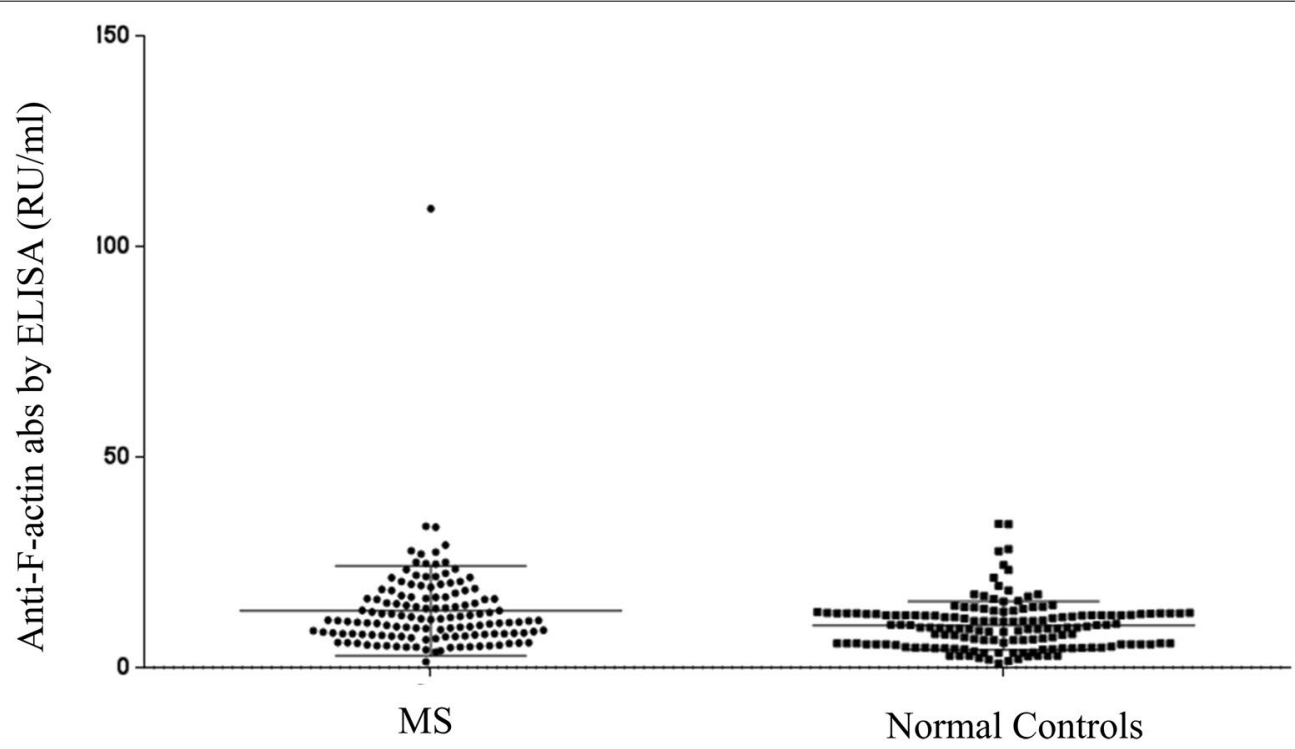

Fig. 3 Levels of anti-F-actin antibodies in patients with multiple sclerosis (MS) and normal controls 


\section{Discussion}

This is the first comprehensive study investigating the presence of AILD-related autoantibodies, both by IIF and antigen-specific assays (line blotting and ELISA) in a large cohort of Greek patients with MS. The major finding of our study is the peculiarly high prevalence of SMA, which rather unexpectedly targets F-actin, the predominant target of AIH-specific SMA with no apparent clinical or other laboratory evidence of underlying $\mathrm{AIH}$. Except SMA, IIF testing reveals autoantibody positivity in a significant proportion of MS patients. However, testing of the seropositive cases using as antigenic substrate the well-defined molecular targets of these autoantibodies reveals only very few positive samples, raising questions as to whether these autoantibodies are of clinical relevance for the identification of suspected cases, who have or will develop AIH over time.

Various autoantibodies unrelated to the disease have been described in patients with MS, most of them are relevant to concomitant autoimmune diseases, such as thyroid autoantibodies. The presence of non-organ specific autoantibodies (NOSA) and in particular SMA and ANA have been reported extensively, at baseline and over time, in the latter case as result of immunomodulatory treatments which provoke autoantibody induction.

Schuller et al. [21] have detected DNA and RNA-specific autoantibodies, both in serum and CSF samples of MS patients. The notion that ANA can also be found in CSF has been challenged by more recent studies [22]. ANA in general, before or after treatment for MS has been reported in a range of $10-81 \%$ [2, 23, 24]. Seyfert et al. found ANA in $10.2 \%$ of MS patients, Heinzlef et al. in $30 \%$, Aisen et al. in 35\% and Dore-Duffy et al. in $81 \%$ of their MS patients $[2,23,24]$. The tremendous difference in positivity range relates to differences of autoantibodies cut-offs points used, sensitivity of the assays, techniques applied, patients cohorts biases (treatment status, older vs younger age cohorts), and other parameters comprehensively discussed over the years. Special attention has been given on whether IFN-b and other immunomodulatory agents can de novo induced NOSAs or whether the presence of ANAs in MS must alarm neurologists and place a clinical suspicion of autoimmune rheumatic disease highly likely. For example, the early study by DoreDuffy et al. [14], which reported $81 \%$ ANA positivity considered an 1:8 dilution cut off for considering autoantibody positivity, 10 times lower than the 1:80 used by most other studies.

Barned et al. reported ANA in 27\% of their RRMS cohort and have provocatively termed them "falsepositive ANA", as only rarely their presence confirms an underlying autoimmune rheumatic disease [25]. That term raised a heated debate as to whether "false positive" ANA can be an established/legitimate term [22-24]. Nonetheless, case studies reporting drug related (mainly due to IFN-b) autoimmune rheumatic diseases (and in particular SLE) led several authors to conclude that unmasking of underlying autoimmune rheumatic disease cannot be taken lightly over the course of MS [26-30]. The same point has been raised very recently for $\mathrm{AIH}$ and other AILDs diagnosed in patients with MS [9].

Several prospective studies have tackled this experimental question, at times testing large cohorts of patients before and after treatment for a significant period of time [15, 31, 32]. Durelli et al. have reported an $8.1 \%$ and $11 \%$, prevalence of NOSAs, respectively before and after IFN-b1b [31]. Verdum et al. have conduced a multi-centre study assessing the dynamics of NOSAs over time in 156 MS patients treated with IFNb1b (Betaferon ${ }^{\circledR}$ ) [15].

While the prevalence of ANA and SMA at baseline was $4.7 \%$ and $1.3 \%$, this increased to $5.8 \%$ and $3.7 \%$, respectively. Baseline autoantibody positivity persisted at during treatment in the great majority of the cases, while those with de novo induction of autoantibodies demonstrated a continual increase over time to reach their maximum levels at 12 months after treatment [14-18, 22, 32-34].

The clinical significance of detectable ANA in relation to MS is highlighted in numerous studies failing to identify a clear association between their presence and disease activity, progression of disease or response to treatment [22]. SMA in serum specimens of MS patients has been known for long. Early and more recent studies have reported SMA in up to $50 \%$ of patients with MS [13], treated or not, remarking their relatively low/moderate levels, i.e. contrasting the SMAs of AIH-1 which can be as high as $1 / 10240$ or higher $[19,35]$.

The novel finding we submit is that these low level SMAs in our cohort are of the SMA-VG (vessel glomeruli) pattern by IIF in kidney rodent tissue, a pattern which is also seen in AIH-1 and are directed against F-actin, the predominant target of AIH-1 specific SMA. We indeed have found by ELISA low titre anti-F-actin abs in 21 (15.8\%), patients with MS (3 of them had also detectable SMA-F pattern by IIF), only 2 of whom had underlying AIH-1, the reference disease for F-actin SMA positivity. Of interest, local synthesis of SMA in the central nervous system has also been reported, but the mechanism for the induction remains unclear [36]. Intriguingly, myelin basic protein (MBP), a major MS-specific autoantigen, binds to negatively charged lipids on the cytosolic surface of oligodendrocyte membranes and can also polymerize actin, bundle F-actin filaments, and bind actin filaments to lipid bilayers through electrostatic interactions [37]. 
Whether MBP-bound F-actin serves as a neoantigen, responsible for the induction of anti-MBP abs but also F-actin SMA in patients with MS remains to be seen.

Finally, our study is probably the first to report on MS the presence of anti-Ro52 abs, an autoantibody marker of prototype autoimmune rheumatic diseases such as systemic lupus erythematosus and Sjögren's syndrome. This autoantibodies, which is relatively frequent and can be found in non-autoimmune rheumatic diseases, as monospecificity (anti-Ro52 antibody positive in the absence of anti-Ro60) [38-42] as only present in $2(1.5 \%)$ MS patients, a prevalence unexpectedly low, given the reported high prevalence of ANA in MS, autoimmune nature of the disease, and the relatively high prevalence of anti-Ro52 abs in the general population; all three points raising the logical expectation of a higher percentage rate for this autoantibody's specificity in MS. None of these two patients had concomitant anti-Ro60 abs or clinical evidence of sicca syndrome.

Rather confusing was the evidence provided regarding other autoantigen specific reactivities, given that such autoantibodies are considered AILD-specific and are infrequently found in other irrelevant autoimmune disease, in the absence of current or future developed disease. Excluding 4 cases with known MS and concomitant AILDs, 7 had shown positivity PBC-associated autoantibodies such as anti-AMA BPO, PBC-specific ANA antigp210, anti-PML and one has shown AIH-2 anti-LKM1 (anti-CYP2D6) abs. The most frequent of those was the presence of anti-PML antibodies, which in PBC giving a characteristic multiple nuclear dot pattern [35, 43-45]. These autoantibodies were found in 6 of our MS patients, however, none of those had $\mathrm{PBC}$ or features compatible with cholestatic liver disease. Their titres were relatively low, significantly lower than those noted in patients with $\mathrm{PBC}$ and none of those had the specific indirect IF pattern, probably due to the fact that their magnitude was low. If the cut off was to be selected at $24 \mathrm{RU} / \mathrm{ml}$ instead of $11 \mathrm{RU} / \mathrm{ml}$ only 2 of those would still be considered positive. Selecting such a cut-off for anti-PML would decrease the sensitivity of the assay for anti-PML from 21 to $17 \%$, as we published in a previous paper in patients with PBC (data not shown) [43]. In case these are no "false-positive" sera, we can only speculate that their presence can be considered as what it has been proposed as a generic a by-product of systemic immune dysregulation noted in MS, that could explain the presence of antiPML and disease-irrelevant ANA [22]. Another tentative explanation would be that related to the ability of IFN- $\beta$ to overexpress nuclear's body PML, raising the possibility this to be a neoantigen exposed to immune system's recognition in patients with MS [46]. If this overexpression becomes more evident under the influence of EBV infection, a known factor overexpressing nuclear's body constituents such as PML remains to be seen [47]. Also, we cannot exclude that anti-PML antibody positivity in MS lacks biologically meaningful relevance, especially since it lacks an association with disease-specific indirect IF patterns of multiple nuclear dots.

In this category may also fall, the one case which has shown low-titre anti-LKM1 antibodies and did not raise any suspicion of $\mathrm{AIH}-2$ or had virological evidence of hepatitis $\mathrm{C}$ virus infection (which at times has detectable anti-LKM1 abs).

In our cohort, 2 patients had history of confirmed co-occurrence autoimmune liver disease (one with autoimmune hepatitis and one with primary biliary cholangitis), while the diagnosis of PBC was confirmed in another one patients with abnormal profile of cholestasis. In the remaining cases, during the follow up period, no other diagnosis of AILDs were established.

Laboratorians but especially neurologists must be aware of these aspects, not only because rarely the presence of these autoantibodies (disease-specific or NOSAs) may indicate an underlying AILD but also because they may not hold any significant relevance and do not need to receive a careful follow-up in the absence of other clinical and laboratory features of AILDs.

\section{Abbreviations \\ Ab: Antibody; AlH: Autoimmune hepatitis; AiLD: Autoimmune liver diseases; AMA: Anti-mitochondrial antibody; ANA: Anti-nuclear antibody; AutoAb: Autoantibody; F-actin: Filamentous actin; IIF: Indirect immunofluorescence; SMA: Smooth muscle autoantibody; MS: Multiple sclerosis; PBC: Primary biliary cholangitis; PSC: Primary sclerosing cholangitis.}

Acknowledgements

We thank Dr. Eirini I. Rigopoulou for critical reading of our manuscript and helpful comments.

\section{Experimental studies involving animals \\ Non applicable.}

\section{Authors' contributions}

ZS, CL, TS, WM: experimental work; CL: statistical analysis; ZS, ED, VT, GH: clinical data acquisition; CL, DPB: original manuscript drafting: DPB: original idea, study design, final drafting of the manuscript. All authors read and approved the final manuscript.

\section{Funding}

Not applicable.

Availability of data and materials

Material and data can be available upon request to the corresponding author.

\section{Ethics approval and consent to participate}

The study was been approved by the local Ethics Committee of the University General Hospital of Larissa, University of Thessaly (4/15-04-2016). Written informed consent was obtained by all participants.

Consent for publication

All authors consented for publication. 


\section{Competing interests}

The authors declare that they have no competing interests.

\section{Author details}

${ }^{1}$ Department of Neurology, University General Hospital of Larissa, Faculty of Medicine, School of Health Sciences, University of Thessaly, Larissa, Greece ${ }^{2}$ Department of Rheumatology and Clinical Immunology, University Genera Hospital of Larissa, Faculty of Medicine, School of Health Sciences, University of Thessaly, Biopolis, Larissa 40500, Greece. ${ }^{3}$ Institute of Experimental Immunology, Affiliated to EUROIMMUN AG, Lubeck, Germany. ${ }^{4}$ Department of Neurology, University of Nicosia, Nicosia, Cyprus.

\section{Received: 7 January 2020 Accepted: 28 March 2020}

\section{Published online: 10 April 2020}

\section{References}

1. De Keyser J. Autoimmunity in multiple sclerosis. Neurology. 1988;38:371-4.

2. Seyfert S, Klapps P, Meisel C, Fischer T, Junghan U. Multiple sclerosis and other immunologic diseases. Acta Neurol Scand. 1990;81:37-42.

3. Kreisler A, de Seze J, Stojkovic T, Delisse B, Combelles M, Verier A, et al. Groupe septentrional d'etude et de recherche sur la Sclerose en P: multiple sclerosis, interferon beta and clinical thyroid dysfunction. Acta Neurol Scand. 2003;107:154-7.

4. Isbister CM, Mackenzie PJ, Anderson D, Wade NK, Oger J. Co-occurrence of multiple sclerosis and myasthenia gravis in British Columbia. Mult Scler. 2003:9:550-3.

5. de Seze J, Canva-Delcambre V, Fajardy I, Delalande S, Stojkovic T, Godet $E$, et al. Autoimmune hepatitis and multiple sclerosis: a coincidental association? Mult Scler. 2005;11:691-3.

6. Pontecorvo MJ, Levinson JD, Roth JA. A patient with primary biliary cirrhosis and multiple sclerosis. Am J Med. 1992;92:433-6.

7. Noseworthy JH, Ebers GC. Primary biliary cirrhosis and multiple sclerosis. Am J Gastroenterol. 1989;84:1584-5.

8. Wong GW, Yeong T, Lawrence D, Yeoman AD, Verma S, Heneghan MA. Concurrent extrahepatic autoimmunity in autoimmune hepatitis: implications for diagnosis, clinical course and long-term outcomes. Liver Int. 2017;37:449-57.

9. Rigopoulou El, Gyftaki S, Arvaniti P, Tsimourtou V, Koukoulis GK, Hadjigeorgiou G, et al. Autoimmune hepatitis in patients with multiple sclerosis: the role of immunomodulatory treatment. Clin Res Hepatol Gastroenterol. 2019;43:e25-32.

10. Salas PAO, Parra CO, Florez CEP, Goez LM, Velez-van-Meerbeke A, Rodriguez JH. Safety liver profile of teriflunomide versus interferon beta in multiple sclerosis: systematic review and indirect comparison metaanalysis. Mult Scler Relat Disord. 2018;26:192-200.

11. Hyypia T, Viander M, Reunanen M, Salmi A. Antibodies to nuclear and smooth muscle antigens in multiple sclerosis and control patients. Acta Neurol Scand. 1982;65:629-35.

12. McMillan SA, Haire M, Middleton D. Antibodies to lymphocytes and smooth muscle in the sera of patients with multiple sclerosis. Clin Immunol Immunopathol. 1980;16:374-85.

13. Nordal GJ, Vandvik B. Evidence of local synthesis of smooth-muscle antibodies in the central nervous system in isolated cases of multiple sclerosis and chronic lymphocytic meningoencephalitis. Scand J Immunol. 1977;6:327-34

14. Dore-Duffy P, Donaldson JO, Rothman BL, Zurier RB. Antinuclear antibodies in multiple sclerosis. Arch Neurol. 1982;39:504-6.

15. Verdun E, Isoardo G, Oggero A, Ferrero B, Ghezzi A, Montanari E, et al. Betaferon Safety Trial Study G: autoantibodies in multiple sclerosis patients before and during IFN-beta $1 \mathrm{~b}$ treatment: are they correlated with the occurrence of autoimmune diseases? J Interferon Cytokine Res. 2002;22:245-55

16. Speciale L, Saresella M, Caputo D, Ruzzante S, Mancuso R, Calvo MG, et al. Serum auto antibodies presence in multiple sclerosis patients treated with beta-interferon 1a and 1b. J Neurovirol. 2000;6(Suppl 2):S57-61.

17. Spadaro M, Amendolea MA, Mazzucconi MG, Fantozzi R, Di Lello R, Zangari $P$, et al. Autoimmunity in multiple sclerosis: study of a wide spectrum of autoantibodies. Mult Scler. 1999;5:121-5.
18. Kivisakk P, Lundahl J, von Heigl Z, Fredrikson S. No evidence for increased frequency of autoantibodies during interferon-beta1b treatment of multiple sclerosis. Acta Neurol Scand. 1998:97:320-3.

19. Liaskos C, Bogdanos DP, Davies ET, Dalekos GN. Diagnostic relevance of anti-filamentous actin antibodies in autoimmune hepatitis. J Clin Pathol. 2007;60:107-8

20. Liaskos C, Bogdanos DP, Rigopoulou El, Dalekos GN. Development of antimitochondrial antibodies in patients with autoimmune hepatitis: art of facts or an artifact? J Gastroenterol Hepatol. 2007:22:454-5.

21. Schuller E, Delasnerie N, Lebon P. DNA and RNA antibodies in serum and CSF of multiple sclerosis and subacute sclerosing panencephalitis patients. J Neurol Sci. 1978;37:31-6.

22. Collard RC, Koehler RP, Mattson DH. Frequency and significance of antinuclear antibodies in multiple sclerosis. Neurology. 1997:49:857-61.

23. Heinzlef O, Johannet C, Tournier-Lasserve E, Roullet E. Antinuclear antibodies in multiple sclerosis. Neurology. 1995:45:2299-300.

24. Aisen ML, Aisen PS. Antinuclear antibodies in multiple sclerosis. Neurology. 1995;45:2299-300.

25. Barned S, Goodman AD, Mattson DH. Frequency of anti-nuclear antibodies in multiple sclerosis. Neurology. 1995:45:384-5.

26. Crispin JC, Diaz-Jouanen E. Systemic lupus erythematosus induced by therapy with interferon-beta in a patient with multiple sclerosis. Lupus. 2005;14:495-6.

27. Kyrozis A, Kararizou E, Georgila E, Loukaidis PN, Koureas A, Rombos A, et al. Rare association of multiple sclerosis and systemic lupus erythematosus. Lupus. 2007;16:991-2

28. Bonaci-Nikolic B, Jeremic I, Andrejevic S, Sefik-Bukilica M, Stojsavljevic $\mathrm{N}$, Drulovic J. Anti-double stranded DNA and lupus syndrome induced by interferon-beta therapy in a patient with multiple sclerosis. Lupus. 2009;18:78-80

29. Sladkova V, Mares J, Lubenova B, Hlustik P, Kanovsky P. Drug-induced systemic lupus erythematosus in interferon beta-1b therapy. Neuro Endocrinol Lett. 2011:32:4-6.

30. Bahri DM, Khiari H, Essouri A, Laadhar L, Zaraa I, Mrabet A, et al. Systemic lupus erythematosus induced by interferon beta1 therapy in a patient with multiple sclerosis. Fundam Clin Pharmacol. 2012:26:210-1.

31. Durelli L, Ferrero B, Oggero A, Verdun E, Ghezzi A, Montanari E, et al. Betaferon Safety Trial Study G: liver and thyroid function and autoimmunity during interferon-beta $1 \mathrm{~b}$ treatment for MS. Neurology. 2001:57:1363-70

32. Polman CH, Kappos L, Dahlke F, Graf R, Beckmann K, Bogumil T, et al. European Study Group on Interferon Beta-1b in S: interferon beta-1 $\mathrm{b}$ treatment does not induce autoantibodies. Neurology. 2005;64(6):996-1000.

33. Fulford KW, Catterall RD, Delhanty JJ, Doniach D, Kremer M. A collagen disorder of the nervous system presenting as multiple sclerosis. Brain. 1972:95:373-86.

34. Pozzilli C, Bastianello S, Gasperini C, Salvetti M. Antinuclear antibodies and MRI activity in multiple sclerosis. Neurology. 1998;51:650.

35. Bogdanos DP, Invernizzi P, Mackay IR, Vergani D. Autoimmune liver serology: current diagnostic and clinical challenges. World J Gastroenterol. 2008;14:3374-87

36. McMillan SA, Haire M. The specificity of IgG- and IgM-class smooth muscle antibody in the sera of patients with multiple sclerosis and active chronic hepatitis. Clin Immunol Immunopathol. 1979;14:256-63.

37. Boggs JM, Rangaraj G, Hill CM, Bates IR, Heng YM, Harauz G. Effect of arginine loss in myelin basic protein, as occurs in its deiminated charge soform, on mediation of actin polymerization and actin binding to a lipid membrane in vitro. Biochemistry. 2005:44:3524-34.

38. Lee AYS. A review of the role and clinical utility of anti-Ro52/TRIM21 in systemic autoimmunity. Rheumatol Int. 2017;37:1323-33.

39. Murng SHK, Thomas M. Clinical associations of the positive anti Ro52 without Ro60 autoantibodies: undifferentiated connective tissue diseases. J Clin Pathol. 2018:71:12-9.

40. Gkoutzourelas A, Liaskos C, Mytilinaiou MG, Simopoulou T, Katsiari C, Tsirogianni A, et al. Anti-Ro60 seropositivity determines Anti-Ro52 Epitope mapping in patients with systemic sclerosis. Front Immunol. 2018;9:2835.

41. Zachou K, Gampeta S, Gatselis NK, Oikonomou K, Goulis J, Manoussakis $M N$, et al. Anti-SLA/LP alone or in combination with anti-Ro52 and fine 
specificity of anti-Ro52 antibodies in patients with autoimmune hepatitis. Liver Int. 2015;35:660-72.

42. Robbins A, Hentzien M, Toquet S, Didier K, Servettaz A, Pham BN, et al. Diagnostic Utility of Separate Anti-Ro60 and Anti-Ro52/TRIM21 Antibody Detection in Autoimmune Diseases. Front Immunol. 2019;10:444.

43. Mytilinaiou MG, Meyer W, Scheper T, Rigopoulou El, Probst C, Koutsoumpas AL, et al. Diagnostic and clinical utility of antibodies against the nuclear body promyelocytic leukaemia and Sp100 antigens in patients with primary biliary cirrhosis. Clin Chim Acta. 2012;413:1211-6.

44. Bogdanos DP, Komorowski L. Disease-specific autoantibodies in primary biliary cirrhosis. Clin Chim Acta. 2011;412:502-12.

45. Bogdanos DP, Baum H, Vergani D. Antimitochondrial and other autoantibodies. Clin Liver Dis. 2003;7:759-77.
46. Janer A, Martin E, Muriel MP, Latouche M, Fujigasaki H, Ruberg M, et al. PML clastosomes prevent nuclear accumulation of mutant ataxin-7 and other polyglutamine proteins. J Cell Biol. 2006;174:65-76.

47. Nicewonger J, Suck G, Bloch D, Swaminathan S. Epstein-Barr virus (EBV) SM protein induces and recruits cellular Sp110b to stabilize mRNAs and enhance EBV lytic gene expression. J Virol. 2004;78:9412-22.

\section{Publisher's Note}

Springer Nature remains neutral with regard to jurisdictional claims in published maps and institutional affiliations.
Ready to submit your research? Choose BMC and benefit from:

- fast, convenient online submission

- thorough peer review by experienced researchers in your field

- rapid publication on acceptance

- support for research data, including large and complex data types

- gold Open Access which fosters wider collaboration and increased citations

- maximum visibility for your research: over $100 \mathrm{M}$ website views per year

At BMC, research is always in progress.

Learn more biomedcentral.com/submissions 